\title{
Comparison between Dual Layer Intraperitoneal Mesh and Single Layer Mesh for Laparoscopic Ventral and Incisional Hernia Repair
}

\author{
Avirneni Akhil1, Vikram Yogish², N. Sivarajan ${ }^{3}$, Himanshi Grover4, \\ Mohammad Wasim ${ }^{5}$, C. Kamalakhannan ${ }^{6}$, Abdul Rahman ${ }^{7}$ \\ 1, 2, 3, 4, 5, 6, 7 Department of General Surgery, SRM Medical College \\ Hospital and Research Centre, Chennai, Tamil Nadu, India.
}

\section{ABSTRACT}

\section{BACKGROUND}

A hernia is the abnormal exit of tissue or an organ, such as the bowel, through the wall of the cavity in which it normally resides. Hernias come in a variety of forms. The abdomen, specifically the groin, is most frequently involved. Incisional hernias and other ventral hernias are common surgical problems. A prosthetic mesh should always be used in ventral hernia repair (VHR). Now, the polypropylene mesh (PPM) has become the prosthetic mesh of choice in the repair of hernias, including inguinal hernia. Newer meshes are introduced, claiming lesser complication rate, but are invariably costlier than Polypropylene mesh (PPM) by 15 - 20 times. In this study we wanted to evaluate the outcomes of intraperitoneal monofilament and dual layer meshes in laparoscopic incisional and ventral hernia repair.

\section{METHODS}

This is a prospective observational study, with a minimum 30 cases for each type of mesh used. All those patients who had undergone ventral and incisional hernia laparoscopic repair at SRM General Hospital, Chennai between June 2018 and 2021 were included in the study. Data was entered in Microsoft excel for analysis, done by using SPSS software version 23.

\section{RESULTS}

Out of 86 hernias which were repaired laparoscopically, PPM was used in 34 (40\%) and composite meshes in $52(60 \%)$ cases. Out of 52 composite meshes, 11 cases (21.2 \%) turned out with complications whereas out of 34 PPMs (11.8\%), 4 cases turned out with complications.

\section{CONCLUSIONS}

With the composite mesh, complications of intraperitoneal PPM (adhesions, inflammation, intestinal fistulisation, sinus formation, seroma and recurrence) may also occur. The difference between the meshes in the occurrence of these complications is not statistically important.

\section{KEY WORDS}

Hernia, Mesh, Surgery, Intraperitoneal Mesh, Laparoscopic Hernia Repair
Corresponding Author: Dr. Avirneni Akhil, H. NO. 2-8, Lakshmi Nagar Colony, Suraram, Jeedimetla, Hyderabad - 500055, Telangana, India.

E-mail: aa1892@srmist.edu.in

DOI: $10.14260 / j e m d s / 2021 / 387$

How to Cite This Article:

Akhil A, Yogish V, Sivarajan, et al. Comparison between dual layer intraperitoneal mesh and single layer mesh for laparoscopic ventral and incisional hernia repair. J Evolution Med Dent Sci 2021;10(25):1871-1875, 10.14260/jemds/2021/387

Submission 11-02-2021,

Peer Review 24-04-2021,

Acceptance 30-04-2021,

Published 21-06-2021.

Copyright (c) 2021 Avirneni Akhil et al. This is an open access article distributed under Creative Commons Attribution License [Attribution 4.0 International (CC BY 4.0)] 


\section{BACKGROUND}

A hernia is defined as an area of weakness or disruption of the fibromuscular tissues of the body wall. It is the abnormal exit of a tissue or an organ through the wall of the cavity in which it typically resides, such as the intestine. With no substantial comorbidities, the presence of a ventral hernia is an indication for repair. The use of prosthetic mesh to repair the defect has decreased the recurrence rates of incisional and ventral hernias. Laparoscopic repair of incisional and ventral hernia with prosthetic mesh is preferred over open repair. ${ }^{1-3}$ The procedure of incisional and ventral hernia laparoscopic repair has almost been standardized. Surgeons are left only with level 2 data i.e. expert opinion, their own experience, hospital buying protocols and contracts with purchasing groups, and recent visits by mesh manufacturing representatives when making decisions on mesh selection for hernia repair.4-5 Surgeon's dilemma is to choose either monofilament polypropylene mesh or newer ones like dual layer meshes.6,7

After the revolution in the surgical treatment of inguinal hernias through anatomic repair, initiated by Edoardo Bassini (1844 - 1924), the surgical treatment of abdominal wall hernias went through a second revolution with the advent of synthetic meshes for reconstruction reinforcement. The principle was to elicit the reinforcement of the region through the production of fibrosis, avoiding the excessive use of tissue tension, which had been considered to be the correct treatment for the repair of hernia defects at that time. René Stoppa (1921 - 2006), one of the pioneers in that revolution, went so far as to state, in 1989, that the definitive surgical cure for any type of hernia was secure. Thereafter, prostheses began to be employed with the objective of reinforcing the abdominal wall, and the use of meshes disseminated rapidly. ${ }^{1}$ The following years did not bring confirmation to that prediction, so attention was redirected to the influence of systemic risk factors and the types of meshes employed. At present, the chapter on abdominal wall repair through synthetic meshes is open once again. ${ }^{2}$

The advent of prosthetic materials, meshes of the most diverse composition, was the key in improving technical outcomes of the surgical repair of congenital or acquired abdominal wall defects. Its utilization, compared with the simple rrhaphy of the aponeurotic defect, reduced the recurrence rates of hernias considerably ${ }^{2-8}$. The polypropylene mesh is the most commonly used material.

VHR is one of the commonest operations performed by general surgeons. It is now proved beyond doubt that primary repair using suture repair techniques should not be used, as they are fallowed, on long term follow up, by unacceptably high recurrence rate, as high as $31 \%-54 \%$. To overcome this high recurrence rate, a prosthetic mesh repair should be used, which decreases the recurrence rate to less than $10 \%{ }^{8}$ Initially steel mesh was used. But it led to problems such as infection, sinus formation, mesh cracking, fragmentation etc. In 1963, Usher introduced PPM in the repair. It has been used in open surgery successfully and is the choice of many surgeons now. However, the mesh is placed in a variety of ways. Techniques of mesh placement include onlay, inlay and sandwich. In the onlay technique, the mesh is placed over the external oblique fascia. In the inlay technique, the mesh is placed either intraperitoneally or in pre peritoneal plane. In the sandwich technique, one mesh is placed onlay and one is placed inlay. Laparoscopic methods are also very popular now and use a mesh which is placed intraperitoneally. A few surgeons do laparoscopic repair by raising a flap of the peritoneum, placing the mesh and closing the peritoneum over the mesh, i.e. separate the mesh and viscera by the peritoneum. ${ }^{2-6}$ Spreading or even stitching / tacking omentum to the mesh to separate it from the viscera is also advocated. However, these techniques may not be always possible as the adequate peritoneum / omentum may not be always available, especially in recurrent hernia cases, because of scarring. Many rents may appear in the peritoneum during dissection. Also, it is technically demanding and all laparoscopic surgeons may not be able to do it. Most of the surgeons place the mesh intraperitoneally, anchoring the mesh with $4-6$ transfascial sutures and tackers on the margins of the mesh in between the sutures to close the gap between the mesh and the abdominal wall, so as to prevent herniation.

If there is a layer of muscle, fascia or peritoneum between the mesh and the viscera, there is no problem. However, if the mesh is in direct contact with intestines, then the question of safety arises. Concerns regarding intraperitoneal PPM are adhesions (with consequent intestinal obstruction), intestinal fistulization, sinus formation and infection. These complications may require surgery to relieve obstruction, removal of the mesh to treat infection or fistula and sometimes even intestinal resection. Mesh removal may be followed by recurrence of hernia. Hence, the newer meshes are introduced, with its attendant high cost. We have a number of newer meshes like polytetrafluoroethylene (PTFE), composite mesh, PCO (polyester coated with antiadhesive collagen layer) etc. When surgeons were asked for their choice of mesh, some of them said that they have been using PPM for a long period without any problem, whereas others were equally sure in saying that PPM should never be used intraperitoneally for the fear of adhesions and fistulization. Most of these decisions were based on anecdotal case reports and undocumented personal experiences. It is desirable to have more scientific data on which we base our choice. We looked into literature for the evidence to accept or reject PPM. This is of great importance as newer meshes are 15 - 20 times costlier than PPM. If PPM can be accepted for intraperitoneal placement, a large number of Indian patients can be benefited by laparoscopic repair, which they cannot afford presently because of high cost of newer meshes.

This research was conducted to assess the effects of the repair of laparoscopic ventral and incisional hernia using monofilament and dual layer intraperitoneal meshes.

\section{METHODS}

This is a prospective observational study wherein a total of 60 patients who had undergone ventral and incisional hernia laparoscopic repair at SRM General Hospital, Chennai between June 2018 and 2021 were taken for the study. Informed consent was taken from the patients before their inclusion in the study. Ethical committee clearance was not obtained since it was an observational study. The data from 
this period was collected retrospectively from the records and reports archived at the Medical records department and also these patients were followed up. All cases were clinically diagnosed with necessary investigations and underwent laparoscopic hernia repair under GA.

Different types of intra peritoneal meshes used for Laparoscopic ventral and incisional hernia repair were Composite / Dual / Bi layer prosthetic mesh (polyurethane on visceral side and polyester on parietal side) and Mono filament polypropylene mesh. The postoperative outcomes were classified as "with complications" and "without complications". The complications included in this study were surgical site wound infection, erosion of the mesh into intestine, adhesions, seroma formation, enterocutaneous fistula, wound sinus, bowel obstructions recurrences, etc. In case of loss to follow up or death, it was taken into account during the statistical analysis. History of previous abdominal surgical operation, the occurrence of surgical site infection, the timing of hernia occurrence and history of associated medical problems (cardiac, respiratory, hepatobiliary, gastrointestinal or endocrinal) were recorded. Abdominal examination focused on the site of the hernia, size of the defect (in the reducible cases), number of defects, association of divarication of recti and its extent.

All patients had a routine preoperative laboratory panel of investigations done that included blood picture, liver function tests (including albumin), kidney functions, blood sugar (fasting) and if elevated further investigations were done with fasting and post prandial blood sugar levels and HbA1c. Electrocardiogram was done in all patients of 35 years or more and in younger patients with a history or clinical signs of cardiac problems. A chest $\mathrm{x}$ ray study and pulmonary function tests were done in all cases recruited during the study period. CT scan of abdomen and pelvis with IV and oral contrast was done (if needed) in all cases to assess the size and width of the defect, calculate the abdominal compartment and hernia sac volume to determine if there was any loss of the domain and to detect any associated intra-abdominal pathology.

\section{Statistical Analysis}

Descriptive statistics comprising mean, average, standard deviation, proportion and percentage was used. Data was entered on Microsoft excel for analysis, done by using SPSS software version 23. Chi-square test was used to arrive at P value.

\section{RESULTS}

\begin{tabular}{|cccc|}
\hline Age Group & Male & Female & Total \\
$21-30$ & 3 & 8 & $\mathbf{1 1}$ \\
$31-40$ & 12 & 20 & $\mathbf{3 2}$ \\
$41-50$ & 11 & 18 & 29 \\
$51-60$ & 6 & 8 & $\mathbf{1 4}$ \\
$61-70$ & 2 & 1 & $\mathbf{3}$ \\
Total & $\mathbf{3 4}(\mathbf{3 8 . 2} \%)$ & $\mathbf{5 5}(\mathbf{6 1 . 8} \%)$ & $\mathbf{8 9}(\mathbf{1 0 0} \%)$ \\
\hline \multicolumn{5}{c}{ Table 1. Participant's Details } \\
\hline
\end{tabular}

\begin{tabular}{|cccc|}
\hline & Composite Mesh \% & PPM \% & Total \% \\
$\begin{array}{c}\text { Without } \\
\text { Complications }\end{array}$ & $41(78.8)$ & $30(88.2)$ & $\mathbf{7 1}(\mathbf{8 2 . 6 )}$ \\
Complications & $11(21.2)$ & $4(11.8)$ & $\mathbf{1 5}(17.4)$ \\
Total & $\mathbf{5 2}(\mathbf{1 0 0 . 0 )}$ & $\mathbf{3 4}(100.0)$ & $\mathbf{8 6}$ \\
\hline Table 2. With and without Complications with the Composite Mesh \\
\hline
\end{tabular}

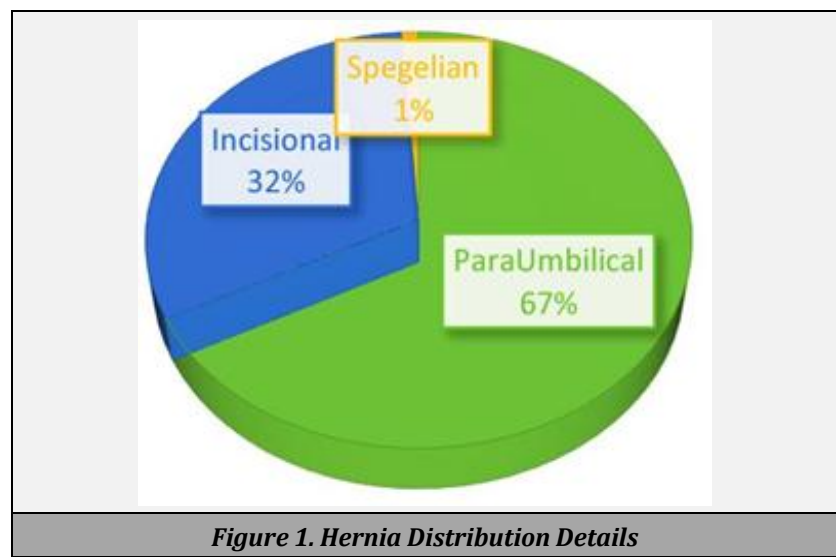

The majority of the patients in the study were in the age group of 31 - 50 years accounting to $68.8 \%$ of total cases. Youngest patient in the study was 28 years old and oldest was 70-year-old. 55 female and 34 male cases were studied.

Para umbilical hernia (67.4 \%) was the most common presentation; following this was incisional hernia (31.4\%) and Spigelian hernia (1.1\%). Out of 89 attempted cases for laparoscopic repair, 3 cases were converted to open repair because of bile leak while undergoing concurrent laparoscopic cholecystectomy (Para umbilical hernia), enterotomy (incisional hernia), and dense adhesions (incisional hernia) on 3 separate occasions. Out of 86 hernias which were repaired laparoscopically, composite meshes were used in 52 (60\%) cases and PPM in 34 (40\%).

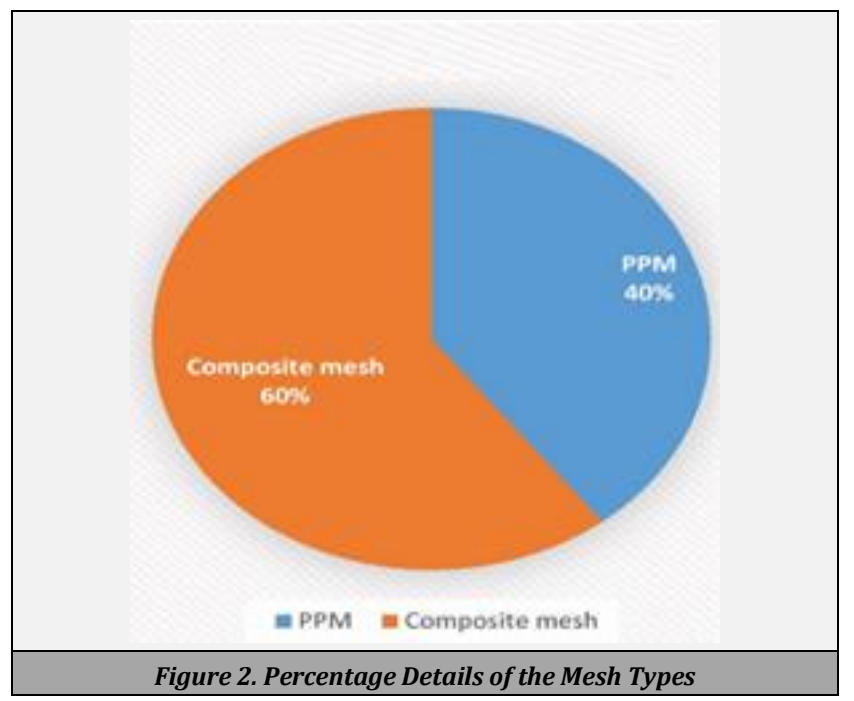

Para umbilical hernia (67.4 \%) was the most common presentation preceded by incisional hernia (31.4\%) and Spigelian hernia (1.1\%).

Average follow up duration for PPM (Polypropylene mesh) repair was 24.2 months and for that of composite mesh repair was 14.6 months. PPM (Polypropylene mesh) repair had $11.8 \%$ and composite mesh repair had $21.2 \%$ complications. We have not come across adhesion formation, bowel obstruction, erosion of mesh into the intestine, entero cutaneous fistula, recurrences, or mortality in our study.

Even though, there are relative risk of complications in composite meshes [RR $=0.79$ (95\% CI: $0.55-1.13)$ ]; it was found to be statistically insignificant $(\mathrm{P}=0.2619)$. The 
method used to arrive at P - value is mentioned in the methods section (chi-square).

\begin{tabular}{|ccccccc|}
\hline Events & PPM & $\mathbf{\%}$ & Composite Mesh & $\mathbf{\%}$ & Total & $\mathbf{\%}$ \\
Seroma & 2 & $(5.9)$ & 3 & $(5.8)$ & 5 & $(5.8)$ \\
Infection & 0 & $(0.0)$ & 2 & $(3.8)$ & 2 & $(2.3)$ \\
Post op ileus & 1 & $(2.9)$ & 2 & $(3.8)$ & 3 & $(3.5)$ \\
Persistent pain & 1 & $(2.9)$ & 3 & $(5.8)$ & 4 & $(4.7)$ \\
Infection \& Sinus & 0 & $(0.0)$ & 1 & $(1.9)$ & 1 & $(1.2)$ \\
Uneventful & 30 & $(88.2)$ & 41 & $(78.8)$ & 71 & $(82.6)$ \\
Total & $\mathbf{3 4}$ & $(\mathbf{1 0 0 . 0})$ & $\mathbf{5 2}$ & $(\mathbf{1 0 0 . 0 )}$ & $\mathbf{8 6}$ & \\
\hline Table 3. Complications Occurred with the Composite Mesh \\
\hline \multicolumn{6}{|c}{} \\
\hline
\end{tabular}

\begin{tabular}{|c|c|c|c|c|c|c|c|}
\hline PPM & 719 & 12 & 1 & 7 & 7 & 21 & 40 \\
\hline Newer Mesh & 1762 & 29 & 2 & 12 & 4 & 76 & 33 \\
\hline P Value & & 0.967 & 1 & 0.452 & 0.018 & 0.117 & $<0.0001$ \\
\hline Significance & & NS & NS & NS & Significant & NS & Significant \\
\hline \multicolumn{8}{|c|}{ Table 4. Comparison Results of PPM and Newer Composite Meshes } \\
\hline
\end{tabular}

The question which was raised with PPM was the formation of postoperative adhesions and seroma. But this is largely based on experimental studies on animal models and there is no first level evidence to reject polypropylene (PPM) mesh.

\section{DISCUSSION}

It can be seen from the Table 3 that there is no statistically significant difference in the incidence of complications between PPM and newer mesh groups except in adhesions and seroma formation. So, it can be safely stated that adhesions and seroma are the only complications to be considered while selecting the mesh. Fear of other complications of PPM mentioned in the literature, is exaggerated. They occur rarely with PPM as well as with newer meshes. High incidence of seroma in PPM group was seen in one study by PK Chowbey ${ }^{9}$ which had contributed to 36 seroma in 40 cases. The author has not given any reason for this high reported incidence of seroma. Barring this one study, none of the other studies showed high incidence of seroma. So, ultimately the only factor to be considered is adhesion formation with consequent risk of intestinal obstruction. In PPM group, out of 7 adhesions, 3 occurred in Nihat yavuz et al. ${ }^{10}$ series and 2 cases in Fuad Alkhouri ${ }^{3}$ series. But none of them required surgery. One case of adhesion of intestines to mesh in Julian Bingener et al.11 series was detected by ultrasound examination and not of any clinical significance. Patient didn't present with clinical intestinal obstruction. Only 1 case in Kua KB et al. ${ }^{12}$ series required re laparoscopy to release the adhesions. Based on these evidences in the literature we conclude that there is no evidence in the published literature to reject PPM for intra peritoneal placement in VHR.

Many of the reports against PPM are based on experimental studies in animal models. JJ Mc Ginty et al. conducted experiments in 8 pigs by placing 3 types of mesh and compared adhesion formation and fibrous ingrowth in 28 days. They found adhesions and adhesion peel strength least with PCO (polyester with anti-adhesive collagen layer) mesh, less with PTFE compared to PPM. PCO facilitates fibrous ingrowth better. In 2009 Nathalie SK reported an experimental study in albine rats comparing PMM with ePTFE mesh which were placed intraperitoneally. They also had a control arm with no mesh placed. All animals killed at
21 days and studied for adhesions, degree of adhesions, percentage of mesh compromised by adhesions and strength needed to rupture these adhesions. Both the groups showed similar results with respect to the factors studied but after excluding adhesions at the edges, ePTFE fared better with respect to adhesions.

\section{CONCLUSIONS}

With the composite mesh, complications of intraperitoneal PPM (adhesions, inflammation, intestinal fistulisation, sinus formation, seroma, and recurrence) may occur. Difference between the meshes in the occurrence of these complications is not statistically significant. We do not even have level 1 evidence in the literature to reject intra peritoneal PPM in VHR. As there is no significant evidence to opine the fallacies involved in the use of monofilament mesh (Polypropylene Mesh) in laparoscopic repair of ventral and incisional hernias (LVIHR), in view of patient's economy it can still be dependent in high volume and low-cost centres where low morbidity due to laparoscopic repair can be of substantial benefit.

Data sharing statement provided by the authors is available with the full text of this article at jemds.com.

Financial or other competing interests: None.

Disclosure forms provided by the authors are available with the full text of this article at jemds.com.

\section{REFERENCES}

[1] Gould J. Laparoscopic versus open hernia repair, advances and controversies in minimally invasive surgery. Surg Clin North Am 2008;88(5):1073-8, vii-viii.

[2] Ramakrishna HK, Lakshman K. Intra peritoneal polypropylene mesh and newer meshes in ventral hernia repair: what ebm says?. Indian J Surg 2013;75(5):34651.

[3] Alkhoury F, Helton S, Ippolito RJ. Cost and clinical outcomes of laparoscopic ventral hernia repair using intraperitoneal nonheavyweight polypropylene mesh. Surg Laparosc Endosc Percutan Tech 2011;21(2):82-5.

[4] Vrijland WW, Jeekel J, Steyerberg EW, et al. Intraperitoneal polypropylene mesh repair of incisional hernia is not associated with enterocutaneous fistula. $\mathrm{Br}$ J Surg 2000;87(3):348-52.

[5] Rosen MJ. Polyester-based mesh for ventral hernia repair: is it safe? Am J Surg 2009;197(3):353-9.

[6] Cobbs WS, Harris JB, Lokey JS, et al. Incisional herniorrhaphy with intraperitoneal composite mesh: a report of 95 cases. Am Surg 2003;69(9):784-7.

[7] Basoglu M, Yildirgan MI, Yilmaz I, et al. Late complications of incisional hernias following prosthetic mesh repair. Acta Chir Belg 2004;104(4):425-8.

[8] William SC. Incisional herniorrhaphy with intra peritoneal composite mesh: a report of 95 cases. Presented at annual meeting southeastern surgical congress. Savannah, Georgia, Feb 2003. 
[9] Chowbey PK, Sharma A, Khullar R, et al. Laparoscopic ventral hernia repair. J Laparoendosc Adv Surg Tech A 2000;10(2):79-84.

[10] Yavuz N, Ipek T, As A, et al. Laparoscopic repair of ventral and incisional hernias: our experience in 150 patients. J Laparoendosc Adv Surg Tech A 2005;15(6):601-5.
[11] Bingener J, Kazantsev GB, Chopra S, et al. Adhesion formation after laparoscopic ventral incisional hernia repair with polypropylene mesh: a study using abdominal ultrasound. JSLS 2004;8(2):127-31.

[12] Kua KB, Coleman M, Martin I, et al. Laparoscopic repair of ventral incisional hernia. ANZ J Surg 2002;72(4):2969. 